\title{
Synthesis of \{323\}-p-Octiphenyls: Orthogonal Functionalization along a Rigid-Rod Scaffold for Refined Supramolecular Architecture
}

\author{
Dawn Ronan, Yoann Baudry, Damien Jeannerat and Stefan Matile* \\ Department of Organic Chemistry, University of Geneva, Geneva, Switzerland.
}

\section{Supporting Information}

General. Reagents for synthesis were purchased either from Aldrich, Fluka or Acros. Solvents were distilled and dried before use. All reactions were performed under an argon atmosphere. Column chromatography was carried out on silica gel 60 (Fluka, 40-63 $\mu \mathrm{m}$ ). Analytical (TLC) and preparative thin layer chromatography (PTLC) were performed on silica gel 60 (Fluka, $0.2 \mathrm{~mm}$ ) and silica gel GF (Analtech, $1000 \mu \mathrm{m}$ ), respectively. IR spectra were recorded on a Perkin Elmer Spectrum One FT-IR spectrometer and are reported in $\mathrm{cm}^{-1}$. Band intensities are indicated as s (strong), $\mathrm{m}$ (medium) and w (weak). ESI-MS and APCI-MS were performed on a Finnigan MAT SSQ 7000 instrument. ${ }^{1} \mathrm{H}$ and ${ }^{13} \mathrm{C}$ NMR spectra were recorded (as indicated) on either a DRX-Bruker $400 \mathrm{MHz}$ or $500 \mathrm{MHz}$ spectrometer and are reported as chemical shifts $(\delta)$ in ppm relative to TMS $(\delta=0)$. Spin multiplicities are reported as singlet $(\mathrm{s})$, doublet (d) or triplet (t), with coupling constants $(J)$ given in $\mathrm{Hz} .{ }^{1} \mathrm{H}$ and ${ }^{13} \mathrm{C}$ NMR resonances were assigned with the aid of additional information from 2D NMR spectra [H, H-COSY (e.g., Figure S1), HSQC, HMBC (e.g., Figure S2)].

NMR experiments carried out for compounds $\mathbf{1 a}$ and $\mathbf{1 b}$ were performed on a Bruker $500{ }^{1} \mathrm{H}$ Larmor frequency spectrometer at room temperature. The DQF-COSY spectra, shown for compound 1a in Figure S1, was acquired using a 1.8 and 8 ppm width in the first and second dimensions respectively. The number of time increments was 256 and 2048 giving a resolution of $2.8 \times 1.4 \mathrm{~Hz} /$ point. In the carbon dimension, the conventional and aliased HMBC spectra were acquired using 256 and 517 time increments respectively. The spectral width of the first was set to the full width $(30188 \mathrm{~Hz}$ ) while the aliased spectrum was $970.214 \mathrm{~Hz}$ after optimization (see Ref. 11c) based on data stemming from a $\left\{{ }^{1} \mathrm{H}\right\}^{13} \mathrm{C}$ spectrum. The resulting digital 
resolutions were 117.92 and $1.87 \mathrm{~Hz} /$ point respectively and acquisition times $2 \mathrm{~h} 10$ $\min$ and $24 \mathrm{~h} 22 \mathrm{~min}$ respectively. The additional experimental time is required due to the molecular diffusion that occurs during the much longer time evolution of the aliased spectrum. The complete aliased spectrum for 1a is given in Fig. S2 and details shown in Fig. 2. HSQC spectra (not shown) have also been acquired using 344 time increments and a spectral width of $325.73 \mathrm{~Hz}$ after optimization of the DEPT spectrum data resulting in a resolution of $0.94 \mathrm{~Hz} /$ point in $1 \mathrm{~h} 23 \mathrm{~min}$. In the proton dimensions, all heteronuclear experiments were acquired with an acquisition time of 0.528 seconds.

4,4'-Diiodo-3,3'-dimethoxybiphenyl (6). As in F. Robert, J.-Y. Winum, N. Sakai, D. Gerard, S. Matile, Org. Lett. 2000, 2, 37, Supporting Information.

4,4'-Diiodo-3,3'-dihydroxybiphenyl (7). As in F. Robert, J.-Y. Winum, N. Sakai, D. Gerard, S. Matile, Org. Lett. 2000, 2, 37, Supporting Information.

Biphenyl dibenzylester 4. To a solution of $7(219 \mathrm{mg}, 0.5 \mathrm{mmol})$ in dry acetone (10 $\mathrm{ml}$ ) was added $\mathrm{Cs}_{2} \mathrm{CO}_{3}(652 \mathrm{mg}, 2.0 \mathrm{mmol})$. The resulting suspension was stirred at room temperature for $30 \mathrm{~min}$ before the addition of benzyl bromoacetate ( $314 \mu 1,2.0$ $\mathrm{mmol}$ ) to the mixture. This suspension was stirred for an additional $3 \mathrm{~h}$ before being diluted with ethyl acetate $(20 \mathrm{ml})$, washed with brine $(20 \mathrm{ml}, 1 \mathrm{x})$, dried over anhydrous $\mathrm{Na}_{2} \mathrm{SO}_{4}$, and concentrated in vacuo. The crude product was vortexed with hexane $(20 \mathrm{ml})$, the solvent filtered off, the product collected and dried in vacuo to give $4(308 \mathrm{mg}, 84 \%)$ as a pure colorless solid. TLC $\left(\mathrm{CH}_{2} \mathrm{Cl}_{2}\right): R_{f} 0.58 ; \mathrm{mp} 142.0$ $143.0{ }^{\circ} \mathrm{C}$; IR: v $3031 \mathrm{w}, 2931 \mathrm{w}, 1887 \mathrm{w}, 1736 \mathrm{~s}, 1582 \mathrm{w}, 1553 \mathrm{~m}, 1468 \mathrm{~m}, 1433 \mathrm{~m}$, $1378 \mathrm{~m}, 1192 \mathrm{~s}, 1077 \mathrm{~s}, 951 \mathrm{~m} ;{ }^{1} \mathrm{H}$ NMR $\left(500 \mathrm{MHz}, \mathrm{CD}_{2} \mathrm{Cl}_{2}\right): \delta 7.82\left(\mathrm{~d},{ }^{3} J=8.0 \mathrm{~Hz}\right.$, $2 \mathrm{H}), 7.33-7.31(\mathrm{~m}, 10 \mathrm{H}), 6.85\left(\mathrm{dd},{ }^{4} J=1.9 \mathrm{~Hz},{ }^{3} J=8.0 \mathrm{~Hz}, 2 \mathrm{H}\right), 6.79\left(\mathrm{~d},{ }^{4} J=1.9 \mathrm{~Hz}\right.$, 2H), 5.23 (s, 4H), 4.70 (s, 4H); ${ }^{13} \mathrm{C}$ NMR (125 MHz, $\left.\mathrm{CD}_{2} \mathrm{Cl}_{2}\right): \delta 168.3$ (s, 2x), 157.4 (s, 2x), 142.2 (s, 2x), 140.5 (d, 2x), 135.7 (s, 2x), 128.9 (d, 4x), 128.8 (d, 2x), 128.7 $(\mathrm{d}, 4 \mathrm{x}), 122.5(\mathrm{~d}, 2 \mathrm{x}), 111.4(\mathrm{~d}, 2 \mathrm{x}), 85.9(\mathrm{~s}, 2 \mathrm{x}), 67.4(\mathrm{t}, 2 \mathrm{x}), 66.7$ (t, 2x); anal. calc. for $\mathrm{C}_{30} \mathrm{H}_{24} \mathrm{O}_{6} \mathrm{I}_{2}$ (734.32): $\mathrm{C}$ 49.07, $\mathrm{H}$ 3.29; found: $\mathrm{C}$ 49.01, H 3.31; MS (APCI, acetone): $\mathrm{m} / z(\%) 734(5)[\mathrm{M}+\mathrm{H}]^{+}, 608(67)[\mathrm{M}+\mathrm{H}-\mathrm{I}]^{+}, 517(100)\left[\mathrm{M}+\mathrm{H}-\mathrm{I}-\mathrm{C}_{7} \mathrm{H}_{7}\right]^{+}$.

$\mathbf{4}^{\prime \prime}$-Iodo-2,2',3"-trimethoxy-p-terphenyl (12). To a solution of 6 (2.8 g, $\left.6.0 \mathrm{mmol}\right)$ in degassed acetone/water $(200 / 40 \mathrm{ml})$ at $50{ }^{\circ} \mathrm{C}$ was added $\mathrm{K}_{2} \mathrm{CO}_{3}(280 \mathrm{mg}, 2.0 \mathrm{mmol})$ and $\mathrm{Pd}\left(\mathrm{PPh}_{3}\right)_{4}(10 \mathrm{~mol} \%)$. 2-Methoxyphenylboronic acid 11 (304 mg, $2.0 \mathrm{mmol}$, Aldrich) was added via syringe-pump to this solution over $8 \mathrm{~h}$. At the end of the reaction the acetone was evaporated, $\mathrm{CH}_{2} \mathrm{Cl}_{2}$ was added $(20 \mathrm{ml})$, and the organic phase washed with brine $(20 \mathrm{ml}, 2 \mathrm{x})$, dried over anhydrous $\mathrm{Na}_{2} \mathrm{SO}_{4}$, and concentrated 
in vacuo. Purification by column chromatography $\left(\mathrm{CH}_{2} \mathrm{Cl}_{2} /\right.$ petroleum ether $\left.1: 1\right)$ gave 12 as a pure colorless solid $(250 \mathrm{mg}, 37 \%$, conversion yield). Unreacted $\mathbf{6}$ was recovered $(2.1 \mathrm{~g})$. TLC $\left(\mathrm{CH}_{2} \mathrm{Cl}_{2} /\right.$ petroleum ether 1:1): $R_{f} 0.45 ; \mathrm{mp} 149.0-150.0{ }^{\circ} \mathrm{C}$; IR: v 2927w, 2833w, 1582w, 1550m, 1460m, 1376m, 1231s, 1027s, 809s, 755s; ${ }^{1} \mathrm{H}$ NMR $\left(500 \mathrm{MHz}, \mathrm{CDCl}_{3}\right): \delta 7.84\left(\mathrm{~d},{ }^{3} J=8.0 \mathrm{~Hz}, 1 \mathrm{H}\right), 7.37\left(\mathrm{td},{ }^{4} J=1.7 \mathrm{~Hz},{ }^{3} J=7.5\right.$ $\mathrm{Hz}, 1 \mathrm{H}), 7.34\left(\mathrm{~d},{ }^{3} J=7.7 \mathrm{~Hz}, 1 \mathrm{H}\right), 7.29\left(\mathrm{dd},{ }^{4} J=1.7 \mathrm{~Hz},{ }^{3} J=7.5 \mathrm{~Hz}, 1 \mathrm{H}\right), 7.21\left(\mathrm{dd},{ }^{4} J\right.$ $\left.=1.6 \mathrm{~Hz},{ }^{3} J=7.7 \mathrm{~Hz}, 1 \mathrm{H}\right), 7.14\left(\mathrm{~d},{ }^{4} J=1.6 \mathrm{~Hz}, 1 \mathrm{H}\right), 7.04\left(\mathrm{~d},{ }^{4} J=2.0 \mathrm{~Hz}, 1 \mathrm{H}\right), 7.03$ $\left(\mathrm{td},{ }^{4} J=1.0 \mathrm{~Hz},{ }^{3} J=7.5 \mathrm{~Hz}, 1 \mathrm{H}\right), 7.01\left(\mathrm{dd},{ }^{4} J=1.0 \mathrm{~Hz},{ }^{3} J=7.5 \mathrm{~Hz}, 1 \mathrm{H}\right), 7.0\left(\mathrm{dd},{ }^{4} J=\right.$ $\left.2.0 \mathrm{~Hz},{ }^{3} \mathrm{~J}=8.0 \mathrm{~Hz}, 1 \mathrm{H}\right), 3.97$ (s, 3H), 3.85 (s, 3H), 3.82 (s, 3H); ${ }^{13} \mathrm{C}$ NMR $(125 \mathrm{MHz}$, $\mathrm{CDCl}_{3}$ ): $\delta 158.3$ (s), 157.4 (s), 157.0 (s), 143.3 (s), 141.1 (s), 139.6 (d), 131.9 (d), 131.5 (d), 128.8 (d), 127.5 (s), 127.2 (s), 121.5 (d), 120.4 (d), 119.2 (d), 111.1 (d), 110.1 (d, 2x), 84.9 (s), 56.4 (q), 55.9 (q), 55.7 (q); MS (APCI, $\mathrm{CH}_{2} \mathrm{Cl}_{2}$ ): m/z (\%) 446 (22) $[\mathrm{M}+\mathrm{H}]^{+}, 432(44)\left[\mathrm{M}+\mathrm{H}-\mathrm{CH}_{3}\right]^{+}, 320$ (100) $[\mathrm{M}+\mathrm{H}-\mathrm{I}]^{+}, 305$ (21) $\left[\mathrm{M}+\mathrm{H}-\mathrm{I}-\mathrm{CH}_{3}\right]^{+}$, $289(45)\left[\mathrm{M}+\mathrm{H}-\mathrm{I}-2\left(\mathrm{CH}_{3}\right)\right]^{+}$.

4"-Iodo-2,2',3"-trihydroxy-p-terphenyl (13). To a solution of 12 (200 mg, 0.45 mmol) in dry $\mathrm{CH}_{2} \mathrm{Cl}_{2}(80 \mathrm{ml})$ was added boron tribromide $(2.69 \mathrm{ml}$ of a $1 \mathrm{M}$ solution in $\mathrm{CH}_{2} \mathrm{Cl}_{2}$ ) at $-78{ }^{\circ} \mathrm{C}$. This solution was allowed to warm to room temperature over $14 \mathrm{~h}$. After this time, $\mathrm{MeOH}(\sim 0.5 \mathrm{ml})$ was added to quench the reaction, the solution washed with brine $(80 \mathrm{ml}, 3 \mathrm{x})$, dried over anhydrous $\mathrm{Na}_{2} \mathrm{SO}_{4}$, and concentrated in vacuo. Purification of the crude product by column chromatography $\left(\mathrm{CH}_{2} \mathrm{Cl}_{2} / \mathrm{MeOH}\right.$ 25:1) gave 13 (168 mg, 92\%) as a pure colorless solid. TLC $\left(\mathrm{CH}_{2} \mathrm{Cl}_{2} / \mathrm{MeOH} 25: 1\right): R_{f}$ 0.2; mp 76.0-77.0 ${ }^{\circ} \mathrm{C}$; IR: v 3364m, 1613w, 1550m, 1472m, 1452m, 1403s, 1322m, $1288 \mathrm{~m}, 1196 \mathrm{~s}, 1174 \mathrm{~s}, 950 \mathrm{~m}, 792 \mathrm{~s}, 747 \mathrm{~s} ;{ }^{1} \mathrm{H}$ NMR (400 MHz, $\left.\mathrm{CD}_{3} \mathrm{OD}\right): \delta 7.72\left(\mathrm{~d},{ }^{3} J\right.$ $=8.2 \mathrm{~Hz}, 1 \mathrm{H}), 7.29\left(\mathrm{~d},{ }^{3} J=7.8 \mathrm{~Hz}, 1 \mathrm{H}\right), 7.27\left(\mathrm{dd},{ }^{4} J=1.8 \mathrm{~Hz},{ }^{3} J=7.5 \mathrm{~Hz}, 1 \mathrm{H}\right), 7.21$ $\left(\mathrm{td},{ }^{4} J=1.8 \mathrm{~Hz},{ }^{3} J=7.5 \mathrm{~Hz}, 1 \mathrm{H}\right), 7.16\left(\mathrm{dd},{ }^{4} \mathrm{~J}=2.0 \mathrm{~Hz},{ }^{3} \mathrm{~J}=7.8 \mathrm{~Hz}, 1 \mathrm{H}\right), 7.14\left(\mathrm{~d},{ }^{4} \mathrm{~J}=\right.$ $1.8 \mathrm{~Hz}, 1 \mathrm{H}), 7.10\left(\mathrm{~d},{ }^{4} \mathrm{~J}=2.0 \mathrm{~Hz}, 1 \mathrm{H}\right), 6.94\left(\mathrm{td},{ }^{4} \mathrm{~J}=1.0 \mathrm{~Hz},{ }^{3} \mathrm{~J}=7.5 \mathrm{~Hz}, 1 \mathrm{H}\right), 6.93(\mathrm{dd}$, $\left.{ }^{4} \mathrm{~J}=1.0 \mathrm{~Hz},{ }^{3} \mathrm{~J}=7.5 \mathrm{~Hz}, 1 \mathrm{H}\right), 6.88\left(\mathrm{dd},{ }^{4} \mathrm{~J}=2.0 \mathrm{~Hz},{ }^{3} \mathrm{~J}=8.2 \mathrm{~Hz}, 1 \mathrm{H}\right) ;{ }^{13} \mathrm{C}$ NMR $(100$ $\mathrm{MHz}, \mathrm{CD}_{3} \mathrm{OD}$ ): $\delta 158.4$ (s), 155.5 (s), 155.1 (s), 143.8 (s), 142.0 (s), 140.6 (d), 133.2 (d), 132.7 (d), 129.8 (d), 127.2 (s, 2x), 121.4 (d), 120.8 (d), 119.7 (d), 117.3 (d), 115.5 (d), 114.1 (d), 83.4 (s); anal. calc. for $\mathrm{C}_{18} \mathrm{H}_{13} \mathrm{O}_{3} \mathrm{I}\left(0.367 \mathrm{H}_{2} \mathrm{O}\right)(410.78): \mathrm{C} 52.63, \mathrm{H}$ 3.37; found: C 52.63, $\mathrm{H} 3.37$; MS (APCI, $\mathrm{CH}_{2} \mathrm{Cl}_{2} / \mathrm{MeOH}$ ): $m / z$ (\%) 807 (30) [2M-H] ${ }^{-}$, $403(100)[\mathrm{M}-\mathrm{H}]^{-}$.

p-Terphenyl tri-tert-butylester 14. To a solution of 13 (152 $\mathrm{mg}, 0.38 \mathrm{mmol})$ in dry acetone $(10 \mathrm{ml})$ was added $\mathrm{Cs}_{2} \mathrm{CO}_{3}(749 \mathrm{mg}, 2.3 \mathrm{mmol})$. The resulting suspension was stirred at room temperature for $30 \mathrm{~min}$ after which time tert-butylbromoacetate (340 $\mu 1,2.3 \mathrm{mmol}$ ) was added. The reaction was stirred for an additional $3 \mathrm{~h}$. The mixture was diluted with ethyl acetate $(20 \mathrm{ml})$, washed with brine $(30 \mathrm{ml}, 1 \mathrm{x})$, dried over 
anhydrous $\mathrm{Na}_{2} \mathrm{SO}_{4}$, and concentrated in vacuo. Purification of the crude product by column chromatography $\left(\mathrm{CH}_{2} \mathrm{Cl}_{2} /\right.$ acetone 100:1) gave $14(256 \mathrm{mg}, 90 \%)$ as a pure colorless solid. TLC $\left(\mathrm{CH}_{2} \mathrm{Cl}_{2} / \mathrm{MeOH} 25: 1\right): R_{f} 0.89 ; \mathrm{mp} 42.0-43.0{ }^{\circ} \mathrm{C}$; IR: v $2978 \mathrm{w}$, $2932 \mathrm{w}, 1750 \mathrm{~m}, 1725 \mathrm{~m}, 1552 \mathrm{~m}, 1474 \mathrm{~m}, 1450 \mathrm{w}, 1393 \mathrm{w}, 1367 \mathrm{~m}, 1214 \mathrm{~m}, 1151 \mathrm{~s}$, $1075 \mathrm{~m}, 810 \mathrm{~m}, 845 \mathrm{~m}, 750 \mathrm{~m} ;{ }^{1} \mathrm{H}$ NMR $\left(500 \mathrm{MHz}, \mathrm{CD}_{3} \mathrm{OD}\right): \delta 7.84\left(\mathrm{~d},{ }^{3} J=7.9 \mathrm{~Hz}\right.$, $1 \mathrm{H}), 7.36\left(\mathrm{~d},{ }^{3} J=7.7 \mathrm{~Hz}, 1 \mathrm{H}\right), 7.32\left(\mathrm{dd},{ }^{4} J=1.7 \mathrm{~Hz},{ }^{3} J=7.4 \mathrm{~Hz}, 1 \mathrm{H}\right), 7.30\left(\mathrm{td},{ }^{4} J=\right.$ $\left.1.7 \mathrm{~Hz},{ }^{3} J=7.4 \mathrm{~Hz}, 1 \mathrm{H}\right), 7.26\left(\mathrm{dd},{ }^{4} J=1.6 \mathrm{~Hz},{ }^{3} J=7.7 \mathrm{~Hz}, 1 \mathrm{H}\right), 7.12\left(\mathrm{~d},{ }^{4} J=1.6 \mathrm{~Hz}\right.$, $1 \mathrm{H}), 7.06\left(\mathrm{~d},{ }^{4} J=2.0 \mathrm{~Hz}, 1 \mathrm{H}\right), 7.04\left(\mathrm{dd},{ }^{4} J=2.0 \mathrm{~Hz},{ }^{3} J=7.9 \mathrm{~Hz}, 1 \mathrm{H}\right), 7.03\left(\mathrm{td},{ }^{4} J=\right.$ $\left.1.0 \mathrm{~Hz},{ }^{3} J=7.4 \mathrm{~Hz}, 1 \mathrm{H}\right), 6.92\left(\mathrm{~d},{ }^{3} J=8.2 \mathrm{~Hz}, 1 \mathrm{H}\right), 4.76(\mathrm{~s}, 2 \mathrm{H}), 4.58(\mathrm{~s}, 2 \mathrm{H}), 4.52(\mathrm{~s}$, $2 \mathrm{H}), 1.48(\mathrm{~s}, 9 \mathrm{H}), 1.44(\mathrm{~s}, 18 \mathrm{H}) ;{ }^{13} \mathrm{C} \mathrm{NMR}\left(125 \mathrm{MHz}, \mathrm{CD}_{3} \mathrm{OD}\right): \delta 170.2(\mathrm{~s}), 170.1(\mathrm{~s})$, 169.5 (s), 158.9 (s), 157.4 (s), 157.1 (s), 143.9 (s), 141.1 (s), 133.4 (d), 132.8 (d), 129.7 (s), 129.0 (s), 122.9 (d), 122.4 (d), 120.8 (d), 113.8 (d), 112.6 (d), 112.2 (d), 86.0 (s), 67.7 (t), 67.5 (t), 28.4 (q), 28.3 (q); anal. calc. for $\mathrm{C}_{36} \mathrm{H}_{43} \mathrm{O}_{9} \mathrm{I}(746.63)$ : C 57.91, H 5.80; found: C 57.85, H 5.86; MS (APCI, $\mathrm{CH}_{2} \mathrm{Cl}_{2} / \mathrm{MeOH}$ ): $\mathrm{m} / z$ (\%) 746 (30) $[\mathrm{M}+\mathrm{H}]^{+}, 690(45)[\mathrm{M}+\mathrm{H}-t \mathrm{Bu}]^{+}, 634(45)[\mathrm{M}+\mathrm{H}-2 t \mathrm{Bu}]^{+}, 579(60)[\mathrm{M}+\mathrm{H}-3 t \mathrm{Bu}]^{+}, 533$ (90) $\left[\mathrm{M}+\mathrm{H}-3 t \mathrm{Bu}-\mathrm{CH}_{2} \mathrm{O}_{2}\right]^{+}, 487$ (40) $\left[\mathrm{M}+\mathrm{H}-3 t \mathrm{Bu}-2 \mathrm{CH}_{2} \mathrm{O}_{2}\right]^{+}, 406(100)[\mathrm{M}+\mathrm{H}-3 t \mathrm{Bu}-$ $\left.\mathrm{CH}_{2} \mathrm{O}_{2}-\mathrm{I}\right]^{+}, 360$ (35) $\left[\mathrm{M}+\mathrm{H}-3 t \mathrm{Bu}-2 \mathrm{CH}_{2} \mathrm{O}_{2}-\mathrm{I}\right]^{+}$.

(Bn/tBu)-\{323\}-p-octiphenyl 1b. To a solution of $14(180 \mathrm{mg}, 0.24 \mathrm{mmol})$ in degassed acetonitrile $(3 \mathrm{ml})$ was added $\mathrm{PdCl}_{2}(\mathrm{dppf})(3 \mathrm{~mol} \%)$, TEA $(234 \mu \mathrm{l}, 1.68$ $\mathrm{mmol}$ ) and 4,4,5,5-tetramethyl-1,3,2-dioxaborolane (pinacolborane) (140 $\mu 1,0.96$ mmol). The solution was heated at $80^{\circ} \mathrm{C}$ for $3 \mathrm{~h}$, after which time benzene $(8 \mathrm{ml})$ was added and the solution washed with water $(10 \mathrm{ml}, 1 \mathrm{x})$, dried over anhydrous $\mathrm{Na}_{2} \mathrm{SO}_{4}$, and concentrated in vacuo. Without further purification, the crude boronate ester $\mathbf{1 0}$ was added to a solution of $4(50 \mathrm{mg}, 68.0 \mu \mathrm{mol}), \mathrm{Pd}\left(\mathrm{PPh}_{3}\right)_{4}(10 \mathrm{~mol} \%)$ and $\mathrm{K}_{2} \mathrm{CO}_{3}(57$ $\mathrm{mg}, 0.4 \mathrm{mmol})$ in degassed DMSO $(4 \mathrm{ml})$. The solution was heated at $90{ }^{\circ} \mathrm{C}$ for $14 \mathrm{~h}$. The reaction was cooled, diluted with $\mathrm{CHCl}_{3}(10 \mathrm{ml})$, the organic phase washed with water $(10 \mathrm{ml}, 3 \mathrm{x})$, dried over anhydrous $\mathrm{Na}_{2} \mathrm{SO}_{4}$, and concentrated in vacuo. Purification of the crude product by PTLC $\left(\mathrm{CH}_{2} \mathrm{Cl}_{2} / \mathrm{MeOH} 100: 1\right.$, double elution) gave $1 \mathrm{~b}$ as a pure colorless solid (56 mg, $48 \%)$. TLC $\left(\mathrm{CH}_{2} \mathrm{Cl}_{2} / \mathrm{MeOH}\right.$ 80:1): $R_{f} 0.61$; ${ }^{1} \mathrm{H}$ NMR $\left(500 \mathrm{MHz}, \mathrm{CD}_{2} \mathrm{Cl}_{2}\right): \delta 7.49\left(\mathrm{~d},{ }^{3} J=7.8 \mathrm{~Hz}, 2 \mathrm{H}, 4^{6}, 5^{5}\right), 7.47\left(\mathrm{~d},{ }^{3} J=7.9 \mathrm{~Hz}\right.$, $\left.2 \mathrm{H}, 3^{5}, 6^{6}\right), 7.45\left(\mathrm{~d},{ }^{3} J=7.9 \mathrm{~Hz}, 2 \mathrm{H}, 2^{6}, 7^{5}\right), 7.40\left(\mathrm{dd},{ }^{4} J=1.8 \mathrm{~Hz},{ }^{3} J=7.4 \mathrm{~Hz}, 2 \mathrm{H}, 1^{6}\right.$, $\left.8^{6}\right), 7.34\left(\mathrm{ddd},{ }^{4} \mathrm{~J}=1.8 \mathrm{~Hz},{ }^{3} \mathrm{~J}=7.4 \mathrm{~Hz},{ }^{3} J=8.4 \mathrm{~Hz}, 2 \mathrm{H}, 1^{4}, 8^{4}\right), 7.31\left(\mathrm{dd},{ }^{4} \mathrm{~J}=1.8 \mathrm{~Hz}\right.$, $\left.{ }^{3} J=7.9 \mathrm{~Hz}, 2 \mathrm{H}, 2^{5}, 7^{6}\right), 7.31\left(\mathrm{dd},{ }^{4} J=1.8 \mathrm{~Hz},{ }^{3} J=7.9 \mathrm{~Hz}, 2 \mathrm{H}, 3^{6}, 6^{5}\right), 7.32-7.32(\mathrm{~m}$, $\left.4 \mathrm{H}, 4^{o-\mathrm{Bn}}, 5^{o-\mathrm{Bn}}\right), 7.31-7.31\left(\mathrm{~m}, 4 \mathrm{H}, 4^{m-\mathrm{Bn}}, 5^{m-\mathrm{Bn}}\right), 7.30-7.30\left(\mathrm{~m}, 4 \mathrm{H}, 4^{p-\mathrm{Bn}}, 5^{p-\mathrm{Bn}}\right), 7.30(\mathrm{dd}$, $\left.{ }^{4} J=1.7 \mathrm{~Hz},{ }^{3} J=7.8 \mathrm{~Hz}, 2 \mathrm{H}, 4^{5}, 5^{6}\right), 7.11\left(\mathrm{~d},{ }^{4} J=1.7 \mathrm{~Hz}, 2 \mathrm{H}, 4^{3}, 5^{2}\right), 7.10\left(\mathrm{~d},{ }^{4} J=1.8\right.$ $\left.\mathrm{Hz}, 2 \mathrm{H}, 2^{3}, 7^{2}\right), 7.09\left(\mathrm{ddd},{ }^{4} J=1.3 \mathrm{~Hz},{ }^{3} J=7.4 \mathrm{~Hz},{ }^{3} J=7.4 \mathrm{~Hz}, 2 \mathrm{H}, 1^{5}, 8^{5}\right), 7.08\left(\mathrm{~d},{ }^{4} J\right.$ $\left.=1.8 \mathrm{~Hz}, 2 \mathrm{H}, 3^{2}, 6^{3}\right), 6.88\left(\mathrm{dd},{ }^{4} J=1.3 \mathrm{~Hz},{ }^{3} J=8.4 \mathrm{~Hz}, 2 \mathrm{H}, 1^{3}, 8^{3}\right), 5.21\left(\mathrm{~s}, 4 \mathrm{H}, 4^{\mathrm{BnCH} 2}\right.$, $\left.5^{\mathrm{BnCH} 2}\right), 4.78\left(\mathrm{~s}, 4 \mathrm{H}, 4^{\mathrm{CH} 2 \mathrm{CO}}, 5^{\mathrm{CH} 2 \mathrm{CO}}\right), 4.57\left(\mathrm{~s}, 4 \mathrm{H}, 2^{\mathrm{CH} 2 \mathrm{CO}}, 7^{\mathrm{CH} 2 \mathrm{CO}}\right), 4.56\left(\mathrm{~s}, 4 \mathrm{H}, 3^{\mathrm{CH} 2 \mathrm{CO}}\right.$, 
$\left.6^{\mathrm{CH} 2 \mathrm{CO}}\right), 4.53\left(\mathrm{~s}, 4 \mathrm{H}, 1^{\mathrm{CH} 2 \mathrm{CO}}, 8^{\mathrm{CH} 2 \mathrm{CO}}\right), 1.48(\mathrm{~s}, 18 \mathrm{H}, 2 \times t$-Bu), $1.47,(\mathrm{~s}, 18 \mathrm{H}, 2 \times t$-Bu), $1.47\left(\mathrm{~s}, 18 \mathrm{H}, 2 \times t\right.$-Bu) ${ }^{13} \mathrm{C}$ NMR $\left(125 \mathrm{MHz}, \mathrm{CD}_{2} \mathrm{Cl}_{2}\right): \delta 169.1\left(\mathrm{~s}, 4^{\mathrm{CO}}, 5^{\mathrm{CO}}\right), 168.4(\mathrm{~s}$, $\left.1^{\mathrm{CO}}, 8^{\mathrm{CO}}\right), 168.2\left(\mathrm{~s}, 2^{\mathrm{CO}}, 7^{\mathrm{CO}}\right), 168.2\left(\mathrm{~s}, 3^{\mathrm{CO}}, 6^{\mathrm{CO}}\right), 156.2\left(\mathrm{~s}, 3^{3}, 6^{2}\right), 156.2\left(\mathrm{~s}, 2^{2}, 7^{3}\right)$, $156.1\left(\mathrm{~s}, 4^{2}, 5^{3}\right), 156.0\left(\mathrm{~s}, 1^{2}, 8^{2}\right), 142.1\left(\mathrm{~s}, 3^{1}, 6^{4}\right), 142.0\left(\mathrm{~s}, 4^{4}, 5^{1}\right), 141.8\left(\mathrm{~s}, 2^{4}, 7^{1}\right)$, $135.8\left(\mathrm{~s}, 4^{i-\mathrm{Bn}}, 5^{i \mathrm{Bn}}\right), 132.7\left(\mathrm{~d}, 4^{6}, 5^{5}\right), 132.6\left(\mathrm{~d}, 2^{6}, 7^{5}\right), 132.5\left(\mathrm{~d}, 3^{5}, 6^{6}\right), 132.2\left(\mathrm{~d}, 1^{6}\right.$, $\left.8^{6}\right), 129.1\left(\mathrm{~d}, 1^{4}, 8^{4}\right), 128.9\left(\mathrm{~d}, 4^{m-\mathrm{Bn}}, 5^{m-\mathrm{Bn}}\right), 128.8\left(\mathrm{~d}, 4^{p-\mathrm{Bn}}, 5^{p-\mathrm{Bn}}\right), 128.7\left(\mathrm{~d}, 4^{o-\mathrm{Bn}}, 5^{o-\mathrm{Bn}}\right)$, $127.7\left(\mathrm{~s}, 1^{1}, 8^{1}\right), 127.5\left(\mathrm{~s}, 2^{1}, 7^{4}\right), 127.3\left(\mathrm{~s}, 4^{1}, 5^{4}\right), 126.8\left(\mathrm{~s}, 3^{4}, 6^{1}\right), 121.5\left(\mathrm{~d}, 1^{5}, 8^{5}\right)$, $120.7\left(\mathrm{~d}, 4^{5}, 5^{6}\right), 120.4\left(\mathrm{~d}, 2^{5}, 7^{6}\right), 120.3\left(\mathrm{~d}, 3^{6}, 6^{5}\right), 112.4\left(\mathrm{~d}, 1^{3}, 8^{3}\right), 111.7\left(\mathrm{~d}, 4^{3}, 5^{2}\right)$, $111.4\left(\mathrm{~d}, 2^{3}, 7^{2}\right), 111.2\left(\mathrm{~d}, 3^{2}, 6^{3}\right), 82.3(\mathrm{~s}, t-\mathrm{Bu}), 82.2(\mathrm{~s}, t-\mathrm{Bu}), 67.2\left(\mathrm{t}, 4^{\mathrm{BnCH} 2}, 5^{\mathrm{BnCH} 2}\right)$, $66.7\left(\mathrm{t}, 2^{\mathrm{CH} 2 \mathrm{CO}}, 7^{\mathrm{CH} 2 \mathrm{CO}}\right), 66.6\left(\mathrm{t}, 1^{\mathrm{CH} 2 \mathrm{CO}}, 8^{\mathrm{CH} 2 \mathrm{CO}}\right), 66.5\left(\mathrm{t}, 3^{\mathrm{CH} 2 \mathrm{CO}}, 6^{\mathrm{CH} 2 \mathrm{CO}}\right), 66.5\left(\mathrm{t}, 4^{\mathrm{CH} 2 \mathrm{CO}}\right.$, $5^{\mathrm{CH} 2 \mathrm{CO}}$ ), $28.2\left(\mathrm{q}, t\right.$-Bu), 28.1 (q, $t$-Bu); MS (ESI, $\mathrm{CH}_{2} \mathrm{Cl}_{2} / \mathrm{MeOH}$ 9/1): $\mathrm{m} / z$ (\%) 1742 (100) $[\mathrm{M}+\mathrm{Na}]^{+}, 883(54)[\mathrm{M}+\mathrm{H}+\mathrm{Na}]^{2+}$.

Biphenyl di-tert-butylester 8. As in F. Robert, J.-Y. Winum, N. Sakai, D. Gerard, S. Matile, Org. Lett. 2000, 2, 37, Supporting Information.

Biphenyl diacid 9. TFA (10 ml) was added to a stirred solution of $\mathbf{8}(165 \mathrm{mg}, 0.25$ mmol) in dry $\mathrm{CH}_{2} \mathrm{Cl}_{2}(10 \mathrm{ml})$. After $1 \mathrm{~h}$ the solution was concentrated in vacuo (additional $\mathrm{CH}_{2} \mathrm{Cl}_{2}$ was added to aid the removal of TFA) to afford 9 (132 $\left.\mathrm{mg}, 96 \%\right)$ as a pure colorless solid. ${ }^{1} \mathrm{H}$ NMR $\left(400 \mathrm{MHz}, \mathrm{CD}_{3} \mathrm{OD}\right): \delta 7.85\left(\mathrm{~d},{ }^{3} J=8.0 \mathrm{~Hz}, 2 \mathrm{H}\right)$, $7.06(\mathrm{~s}, 2 \mathrm{H}), 7.01\left(\mathrm{~d},{ }^{3} \mathrm{~J}=8.0 \mathrm{~Hz}, 2 \mathrm{H}\right), 4.81(\mathrm{~s}, 4 \mathrm{H})$.

Biphenyl di-p-methoxybenzylester 3. To a solution of $9(139 \mathrm{mg}, 0.25 \mathrm{mmol})$ in DMF ( $3 \mathrm{ml}$ ) was added $N$-hydroxybenzotriazole (HOBt, $101 \mathrm{mg}, 0.75 \mathrm{mmol}$ ), $\mathrm{N}$-(3dimethylaminopropyl)- $N$ '-ethylcarbodiimide hydrochloride (EDC, $144 \mathrm{mg}, 0.75$ mmol) and $p$-methoxybenzyl alcohol $(75 \mu \mathrm{l}, 0.6 \mathrm{mmol})$. The mixture was stirred for $14 \mathrm{~h}, \mathrm{CH}_{2} \mathrm{Cl}_{2}(10 \mathrm{ml})$ was added and the organic phase washed with brine $(10 \mathrm{ml}, 3 \mathrm{x})$, dried over anhydrous $\mathrm{Na}_{2} \mathrm{SO}_{4}$, and concentrated in vacuo. Purification of the crude product by column chromatography $\left(\mathrm{CH}_{2} \mathrm{Cl}_{2} / \mathrm{MeOH} 10: 1\right)$ gave $\mathbf{3}(140 \mathrm{mg}, 70 \%)$ as a pure colorless solid. TLC $\left(\mathrm{CH}_{2} \mathrm{Cl}_{2}\right): R_{f} 0.6 ;{ }^{1} \mathrm{H}$ NMR $\left(400 \mathrm{MHz}\right.$, DMSO- $\left.d_{6}\right): \delta 7.82$ $\left(\mathrm{d},{ }^{3} \mathrm{~J}=8.1 \mathrm{~Hz}, 2 \mathrm{H}\right), 7.27\left(\mathrm{~d},{ }^{3} \mathrm{~J}=8.7 \mathrm{~Hz}, 4 \mathrm{H}\right), 7.07\left(\mathrm{~d},{ }^{4} J=1.8,2 \mathrm{H}\right), 6.98\left(\mathrm{dd},{ }^{4} J=\right.$ $\left.1.8 \mathrm{~Hz},{ }^{3} J=8.1 \mathrm{~Hz}, 2 \mathrm{H}\right), 6.84\left(\mathrm{~d},{ }^{3} J=8.7 \mathrm{~Hz}, 4 \mathrm{H}\right), 5.13(\mathrm{~s}, 4 \mathrm{H}), 5.06(\mathrm{~s}, 4 \mathrm{H}), 3.73$ (s, $6 \mathrm{H})$.

(pMeOBn/tBu)-\{323\}-p-octiphenyl 1a. To a solution of $3(51 \mathrm{mg}, 64.0 \mu \mathrm{mol})$ in degassed DMSO (4 ml) was added $\mathrm{Pd}\left(\mathrm{PPh}_{3}\right)_{4}(10 \mathrm{~mol} \%), \mathrm{K}_{2} \mathrm{CO}_{3}(53 \mathrm{mg}, 0.38 \mathrm{mmol})$ and the crude boronate ester $\mathbf{1 0}(0.16 \mathrm{mmol}$, prepared as described for $\mathbf{1 b})$. The solution was heated at $90{ }^{\circ} \mathrm{C}$ for $14 \mathrm{~h}$. Then, the reaction was cooled, diluted with $\mathrm{CHCl}_{3}(10 \mathrm{ml})$, the organic phase washed with water $(10 \mathrm{ml}, 3 \mathrm{x})$, dried over 
anhydrous $\mathrm{Na}_{2} \mathrm{SO}_{4}$, and concentrated in vacuo. Purification of the crude product by PTLC $\left(\mathrm{CH}_{2} \mathrm{Cl}_{2} / \mathrm{MeOH}\right.$ 100:1, double elution) gave 1a as a pure colorless solid (28 $\mathrm{mg}, 25 \%$, unoptimized, see 1b). TLC $\left(\mathrm{CH}_{2} \mathrm{Cl}_{2} / \mathrm{MeOH} 80: 1\right): R_{f} 0.42 ;{ }^{1} \mathrm{H}$ NMR (500 $\left.\mathrm{MHz}, \mathrm{CD}_{2} \mathrm{Cl}_{2}\right): \delta 7.48\left(\mathrm{~d},{ }^{3} J=7.8 \mathrm{~Hz}, 2 \mathrm{H}, 4^{6}, 5^{5}\right), 7.47\left(\mathrm{~d},{ }^{3} J=7.9 \mathrm{~Hz}, 2 \mathrm{H}, 3^{5}, 6^{6}\right)$, $7.45\left(\mathrm{~d},{ }^{3} J=7.9 \mathrm{~Hz}, 2 \mathrm{H}, 2^{6}, 7^{5}\right), 7.40\left(\mathrm{dd},{ }^{4} J=1.5 \mathrm{~Hz},{ }^{3} J=7.8 \mathrm{~Hz}, 2 \mathrm{H}, 1^{6}, 8^{6}\right), 7.34$ $\left(\mathrm{ddd},{ }^{4} J=1.5 \mathrm{~Hz},{ }^{3} J=7.8 \mathrm{~Hz},{ }^{3} J=8.0 \mathrm{~Hz}, 2 \mathrm{H}, 1^{4}, 8^{4}\right), 7.32\left(\mathrm{dd},{ }^{4} J=2.0 \mathrm{~Hz},{ }^{3} J=7.9\right.$ $\left.\mathrm{Hz}, 2 \mathrm{H}, 2^{5}, 7^{6}\right), 7.32\left(\mathrm{dd},{ }^{4} J=2.0 \mathrm{~Hz},{ }^{3} J=7.9 \mathrm{~Hz}, 2 \mathrm{H}, 3^{6}, 6^{5}\right), 7.29\left(\mathrm{dd},{ }^{4} J=2.0 \mathrm{~Hz},{ }^{3} J\right.$ $\left.=7.8 \mathrm{~Hz}, 2 \mathrm{H}, 4^{5}, 5^{6}\right), 7.25\left(\mathrm{~d},{ }^{3} J=8.9 \mathrm{~Hz}, 4 \mathrm{H}, 4^{o-\mathrm{Bn}}, 5^{o-\mathrm{Bn}}\right), 7.13\left(\mathrm{~d},{ }^{4} J=2.0 \mathrm{~Hz}, 2 \mathrm{H}, 2^{3}\right.$, $\left.7^{2}\right), 7.12\left(\mathrm{~d},{ }^{4} \mathrm{~J}=2.0 \mathrm{~Hz}, 2 \mathrm{H}, 4^{3}, 5^{2}\right), 7.10\left(\mathrm{~d},{ }^{4} J=2.0 \mathrm{~Hz}, 2 \mathrm{H}, 3^{2}, 6^{3}\right), 7.09\left(\mathrm{ddd},{ }^{4} J=\right.$ $\left.1.5 \mathrm{~Hz},{ }^{3} J=7.8 \mathrm{~Hz},{ }^{3} J=7.8 \mathrm{~Hz}, 2 \mathrm{H}, 1^{5}, 8^{5}\right), 6.89\left(\mathrm{dd},{ }^{4} J=1.5 \mathrm{~Hz},{ }^{3} J=8.0 \mathrm{~Hz}, 2 \mathrm{H}, 1^{3}\right.$, $\left.8^{3}\right), 6.83\left(\mathrm{~d},{ }^{3} \mathrm{~J}=8.9 \mathrm{~Hz}, 4 \mathrm{H}, 4^{m-\mathrm{Bn}}, 5^{m-\mathrm{Bn}}\right), 5.15\left(\mathrm{~s}, 4 \mathrm{H}, 4^{\mathrm{BnCH} 2}, 5^{\mathrm{BnCH} 2}\right), 4.74(\mathrm{~s}, 4 \mathrm{H}$, $\left.4^{\mathrm{CH} 2 \mathrm{CO}}, 5^{\mathrm{CH} 2 \mathrm{CO}}\right), 4.57\left(\mathrm{~s}, 4 \mathrm{H}, 2^{\mathrm{CH} 2 \mathrm{CO}}, 7^{\mathrm{CH} 2 \mathrm{CO}}\right), 4.56\left(\mathrm{~s}, 4 \mathrm{H}, 3^{\mathrm{CH} 2 \mathrm{CO}}, 6^{\mathrm{CH} 2 \mathrm{CO}}\right), 4.53(\mathrm{~s}, 4 \mathrm{H}$, $\left.1^{\mathrm{CH} 2 \mathrm{CO}}, 8^{\mathrm{CH} 2 \mathrm{CO}}\right), 3.73\left(\mathrm{~s}, 6 \mathrm{H}, 4^{\mathrm{OCH} 3-\mathrm{Bn}}, 5^{\mathrm{OCH} 3-\mathrm{Bn}}\right), 1.48(\mathrm{~s}, 18 \mathrm{H}, 2 \mathrm{x} t$-Bu$), 1.48(\mathrm{~s}, 18 \mathrm{H}, 2 \mathrm{x}$ $t$ - $\mathrm{Bu}), 1.47\left(\mathrm{~s}, 18 \mathrm{H}, 2 \times t\right.$-Bu); ${ }^{13} \mathrm{C}$ NMR $\left(125 \mathrm{MHz}, \mathrm{CD}_{2} \mathrm{Cl}_{2}\right): \delta 169.2\left(\mathrm{~s}, 4^{\mathrm{CO}}, 5^{\mathrm{CO}}\right)$, $168.4\left(\mathrm{~s}, 1^{\mathrm{CO}}, 8^{\mathrm{CO}}\right), 168.2\left(\mathrm{~s}, 2^{\mathrm{CO}}, 7^{\mathrm{CO}}\right), 168.2\left(\mathrm{~s}, 3^{\mathrm{CO}}, 6^{\mathrm{CO}}\right), 160.2\left(\mathrm{~s}, 4^{p-\mathrm{Bn}}, 5^{p-\mathrm{Bn}}\right), 156.2$ $\left(\mathrm{s}, 2^{2}, 7^{3}\right), 156.2\left(\mathrm{~s}, 3^{3}, 6^{2}\right), 156.2\left(\mathrm{~s}, 4^{2}, 5^{3}\right), 156.0\left(\mathrm{~s}, 1^{2}, 8^{2}\right), 142.1\left(\mathrm{~s}, 3^{1}, 6^{4}\right), 142.0(\mathrm{~s}$, $\left.4^{4}, 5^{1}\right), 141.9\left(\mathrm{~s}, 2^{4}, 7^{1}\right), 132.7\left(\mathrm{~d}, 4^{6}, 5^{5}\right), 132.6\left(\mathrm{~d}, 2^{6}, 7^{5}\right), 132.6\left(\mathrm{~d}, 3^{5}, 6^{6}\right), 132.3(\mathrm{~d}$, $\left.1^{6}, 8^{6}\right), 130.6\left(\mathrm{~d}, 4^{0-\mathrm{Bn}}, 5^{o-\mathrm{Bn}}\right), 129.1\left(\mathrm{~d}, 1^{4}, 8^{4}\right), 127.9\left(\mathrm{~s}, 4^{i-\mathrm{Bn}}, 5^{i \text {-Bn }}\right), 127.7\left(\mathrm{~s}, 1^{1}, 8^{1}\right)$, $127.5\left(\mathrm{~s}, 2^{1}, 7^{4}\right), 127.3\left(\mathrm{~s}, 4^{1}, 5^{4}\right), 126.9\left(\mathrm{~s}, 3^{4}, 6^{1}\right), 121.5\left(\mathrm{~d}, 1^{5}, 8^{5}\right), 120.7\left(\mathrm{~d}, 4^{5}, 5^{6}\right)$, $120.4\left(\mathrm{~d}, 2^{5}, 7^{6}\right), 120.4\left(\mathrm{~d}, 3^{6}, 6^{5}\right), 114.3\left(\mathrm{~d}, 4^{m-\mathrm{Bn}}, 5^{m-\mathrm{Bn}}\right), 112.4\left(\mathrm{~d}, 1^{3}, 8^{3}\right), 111.7\left(\mathrm{~d}, 4^{3}\right.$, $\left.5^{2}\right), 111.5\left(\mathrm{~d}, 2^{3}, 7^{2}\right), 111.3\left(\mathrm{~d}, 3^{2}, 6^{3}\right), 82.3(\mathrm{~s}, t-\mathrm{Bu}), 67.1\left(\mathrm{t}, 4^{\mathrm{BnCH} 2}, 5^{\mathrm{BnCH} 2}\right), 66.7(\mathrm{t}$, $\left.2^{\mathrm{CH} 2 \mathrm{CO}}, 7^{\mathrm{CH} 2 \mathrm{CO}}\right), 66.6\left(\mathrm{t}, 1^{\mathrm{CH} 2 \mathrm{CO}}, 8^{\mathrm{CH} 2 \mathrm{CO}}\right), 66.6\left(\mathrm{t}, 3^{\mathrm{CH} 2 \mathrm{CO}}, 6^{\mathrm{CH} 2 \mathrm{CO}}\right), 66.5\left(\mathrm{t}, 4^{\mathrm{CH} 2 \mathrm{CO}}, 5^{\mathrm{CH} 2 \mathrm{CO}}\right)$, $55.6\left(\mathrm{t}, 4^{\text {оснз-Вn }}, 5^{\text {ОСнз-вn }}\right), 28.2$ (q, $t$-Bu); MS (ESI, $\mathrm{CH}_{2} \mathrm{Cl}_{2} / \mathrm{MeOH}$ 9/1): $\mathrm{m} / z$ (\%) 1803 (100) $[\mathrm{M}+\mathrm{Na}]^{+}, 913(100)[\mathrm{M}+2 \mathrm{Na}]^{2+}$. 


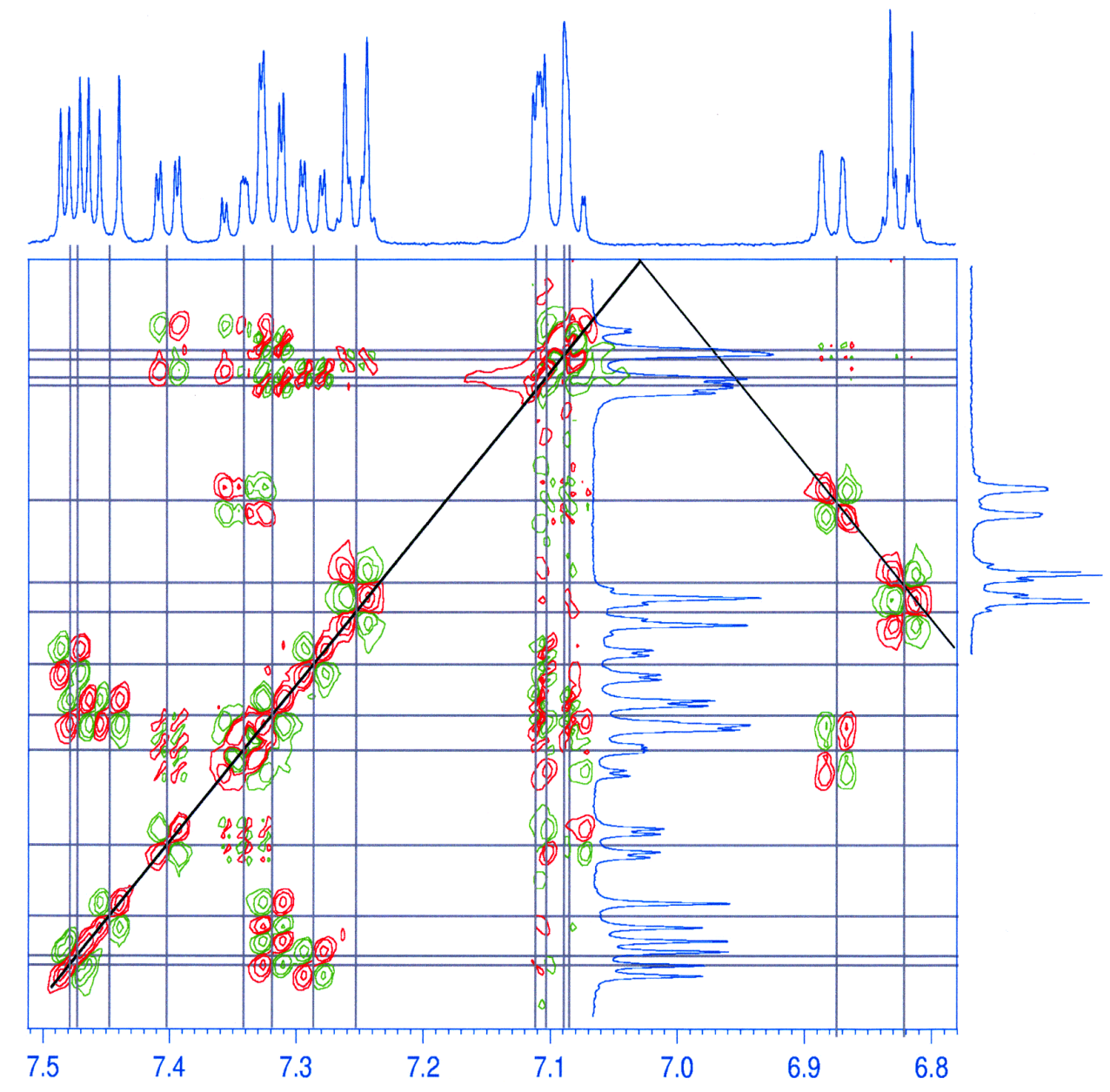

Figure S1. Aromatic regions of a folded DQF-COSY spectrum of 1a: In the first dimension, spectral folding allows one to reach high resolution in less time increments than using the full spectral width but causes the diagonal to zig-zag. In the direct dimension, high resolution is obtained using a large number of data points (4k points). The high resolution is needed to correlate, through the weak ${ }^{4} J$ coupling constant, the aromatic protons that are not direct neighbors. 


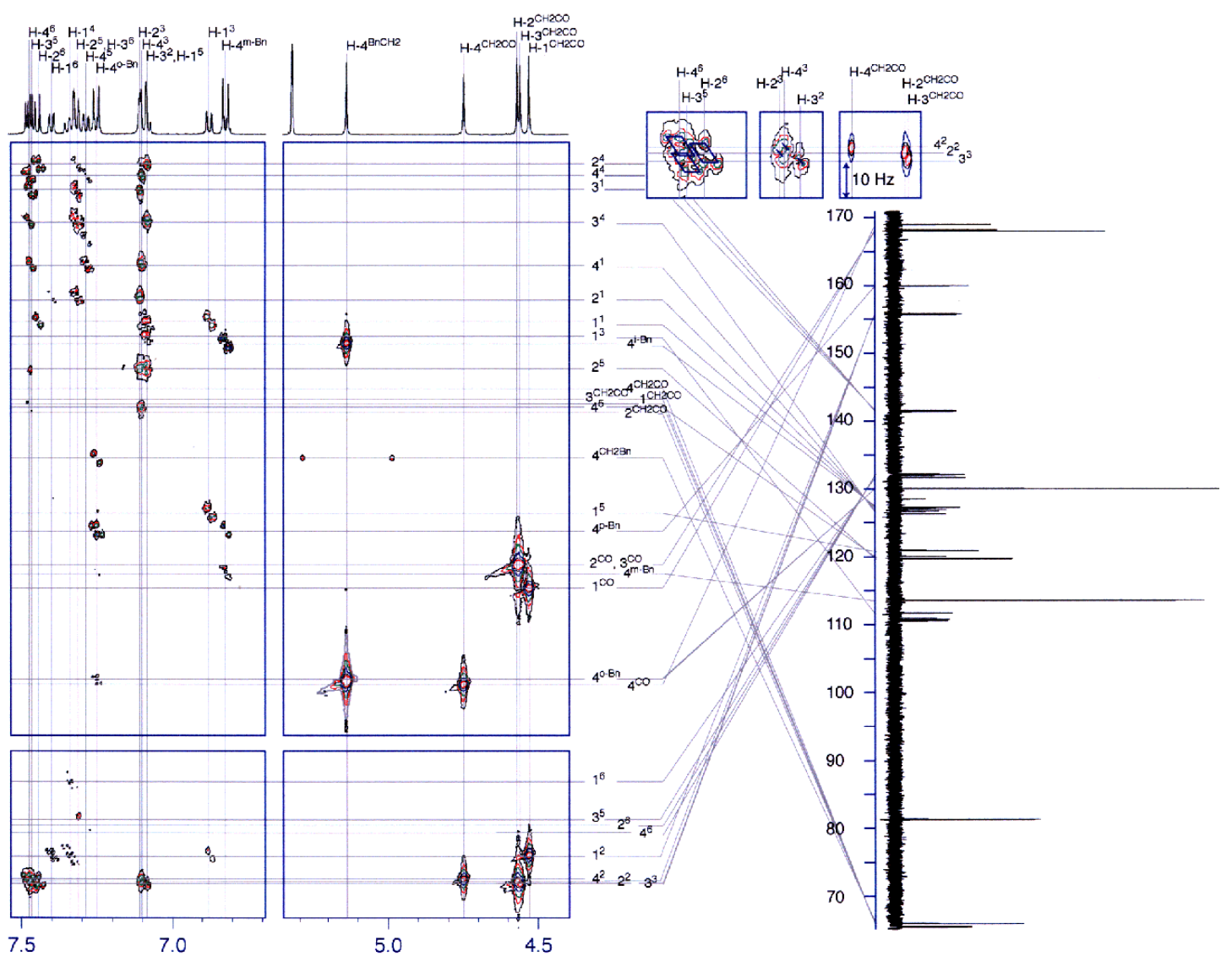

Figure S2. High resolution aliased HMBC spectrum of 1a: Four regions of the same aliased HMBC are shown together with the ${ }^{1} \mathrm{H}$ and ${ }^{13} \mathrm{C}$ NMR spectra of 1a. The most difficult regions are enlarged (top right, and Fig. 2). The fine structure of each signal allows reliable assignment of spin pairs even when signals partially overlap. The broken lines running from the carbon spectrum to the HMBC reflect the change of scale in the first dimension of aliased spectra. 